\title{
Cognitive load theory in health professional education: design principles and strategies
}

Citation for published version (APA):

van Merrienboer, J. J. G., \& Sweller, J. (2010). Cognitive load theory in health professional education: design principles and strategies. Medical Education, 44(1), 85-93. https://doi.org/10.1111/j.13652923.2009.03498.x

Document status and date:

Published: 01/01/2010

DOI:

10.1111/j.1365-2923.2009.03498.x

Document Version:

Publisher's PDF, also known as Version of record

Document license:

Taverne

\section{Please check the document version of this publication:}

- A submitted manuscript is the version of the article upon submission and before peer-review. There can be important differences between the submitted version and the official published version of record.

People interested in the research are advised to contact the author for the final version of the publication, or visit the DOI to the publisher's website.

- The final author version and the galley proof are versions of the publication after peer review.

- The final published version features the final layout of the paper including the volume, issue and page numbers.

Link to publication

\footnotetext{
General rights rights.

- You may freely distribute the URL identifying the publication in the public portal. please follow below link for the End User Agreement:

www.umlib.nl/taverne-license

Take down policy

If you believe that this document breaches copyright please contact us at:

repository@maastrichtuniversity.nl

providing details and we will investigate your claim.
}

Copyright and moral rights for the publications made accessible in the public portal are retained by the authors and/or other copyright owners and it is a condition of accessing publications that users recognise and abide by the legal requirements associated with these

- Users may download and print one copy of any publication from the public portal for the purpose of private study or research.

- You may not further distribute the material or use it for any profit-making activity or commercial gain

If the publication is distributed under the terms of Article $25 \mathrm{fa}$ of the Dutch Copyright Act, indicated by the "Taverne" license above, 


\section{Cognitive load theory in health professional education: design principles and strategies}

Jeroen J G van Merriënboer ${ }^{1,2} \&$ John Sweller ${ }^{3}$

CONTEXT Cognitive load theory aims to develop instructional design guidelines based on a model of human cognitive architecture. The architecture assumes a limited working memory and an unlimited long-term memory holding cognitive schemas; expertise exclusively comes from knowledge stored as schemas in long-term memory. Learning is described as the construction and automation of such schemas. Three types of cognitive load are distinguished: intrinsic load is a direct function of the complexity of the performed task and the expertise of the learner; extraneous load is a result of superfluous processes that do not directly contribute to learning, and germane load is caused by learning processes that deal with intrinsic cognitive load.

OBJECTIVES This paper discusses design guidelines that will decrease extraneous load, manage intrinsic load and optimise germane load.
DISCUSSION Fifteen design guidelines are discussed. Extraneous load can be reduced by the use of goal-free tasks, worked examples and completion tasks, by integrating different sources of information, using multiple modalities, and by reducing redundancy. Intrinsic load can be managed by simple-tocomplex ordering of learning tasks and working from low- to high-fidelity environments. Germane load can be optimised by increasing variability over tasks, applying contextual interference, and evoking self-explanation. The guidelines are also related to the expertise reversal effect, indicating that design guidelines for novice learners are different from guidelines for more experienced learners. Thus, well-designed instruction for novice learners is different from instruction for more experienced learners. Applications in health professional education and current research lines are discussed.

\footnotetext{
${ }^{1}$ Department of Educational Development and Research, Maastricht University, Maastricht,

The Netherlands

${ }^{2}$ CELSTEC, Open University of the Netherlands, Heerlen, The Netherlands

${ }^{3}$ School of Education, University of New South Wales, Sydney,

New South Wales, Australia
}

Correspondence: Jeroen J G van Merriënboer, Maastricht University, FHML, Department of Educational Development and Research, PO Box 616, 6200 MD Maastricht, The Netherlands. Tel: 003143 3885727; Fax: 003143 3885779;

E-mail: j.vanmerrienboer@educ.unimaas.nl 


\section{INTRODUCTION}

Cognitive load theory (CLT) was initially developed in the 1980s. ${ }^{1}$ By means of strictly controlled experimental studies, it aimed to develop instructional design principles and strategies based on a model of human cognitive architecture. Right from the start, CLT provided instructional design principles that were seen as counterintuitive by many practitioners in the field of education. For example, the recommendation to provide novice learners with many worked examples rather than problems to solve contradicted the prevailing opinion of the time that solving problems was the best way to learn to solve problems. The theory has developed substantially since the 1980s (see reviews ${ }^{2,3}$ ). Having established a variety of basic instructional designs that prevented the application of cognitive resources to unnecessary aspects of a task, an increasing number of cognitive load theorists from around the world considered how to deal with highly complex learning tasks, how to stimulate learners to actually use available cognitive resources for learning, and how to deal with the growing expertise of learners in educational programmes of longer duration.

Applications of CLT in medical education are beginning to appear. ${ }^{4,5}$ Moreover, educational approaches in the health professions increasingly stress the use of authentic tasks, which are expected to help students integrate the knowledge, skills and attitudes necessary for effective task performance in professional life. ${ }^{6}$ Authentic learning tasks designed on the basis of real-life tasks have many different solutions, are ecologically valid and usually cannot be mastered in a single session. Whereas CLT may not be relevant to teaching simple tasks, it is critical when complex learning tasks are used because they impose a high load on the learner's cognitive system. ${ }^{7,8}$ Therefore, this article argues that design guidelines based on CLT are highly relevant to teaching in complex domains like the health professions, especially when authentic learning tasks are part of the curriculum. The article briefly presents, in order, the cognitive architecture assumed by CLT, the main design principles and strategies based on this architecture, and a discussion of the presented framework.

\section{COGNITIVE ARCHITECTURE}

Cognitive load theory assumes a cognitive architecture that is broadly supported by research in cognitive psychology and can be explained readily from an evolutionary perspective. ${ }^{9}$ This section briefly summarises the theory in terms of memory systems, learning processes supported by those systems, and associated types of cognitive load.

\section{Memory systems}

Cognitive load theory assumes that the human cognitive system has a limited working memory that can hold no more than five to nine information elements (the famous 'seven plus or minus two') ${ }^{10}$ and actively process no more than two to four elements simultaneously. It is able to deal with information for no more than a few seconds and almost all information is lost after about 20 seconds unless it is refreshed by rehearsal. The theory emphasises that these working memory capacity and duration limitations only apply to novel information obtained through sensory memory. Working memory has no known limitations when dealing with information retrieved from long-term memory. In effect, long-term memory alters the characteristics of working memory. Long-term memory holds cognitive schemas that vary in their degree of complexity and automation. Human expertise comes from knowledge organised by these schemas, not from an ability to engage in reasoning with many elements that have not been organised in long-term memory. Human working memory simply is not able to process many elements.

Expertise develops as learners mindfully combine simple ideas into more complex ones. A medical student, for example, gradually combines simple ideas about consequences, enabling conditions and faults into so-called 'illness scripts', a term that describes a particular type of schema which allows the user to distinguish between alike diseases. Illness scripts can be interpreted during problem solving and reasoning and so help to reach an accurate diagnosis. These schemas organise knowledge but also heavily reduce working memory load because even a highly complex schema can be dealt with as one element in working memory.

Fully automated schemas are developed as a function of extensive practice and can act as a central executive, organising information or knowledge that needs to be processed in working memory. Under these circumstances there are no limits to working memory. For instance, an experienced medical doctor recognises a 'warm shock' resulting from redistribution of cardiac output at a single glance. By contrast, when dealing with novel information for 
which no schema-based central executive is available, working memory has limitations. Thus, for a novice student, a patient with a warm shock may show little more than an unstructured set of symptoms.

\section{Learning processes}

Working memory must inevitably be limited in capacity when dealing with completely novel, unorganised information because, as the number of elements that need to be organised increases linearly, the number of possible combinations of elements that must be tested for effectiveness during problem solving increases exponentially. Random testing of the effectiveness of possible combinations based on many elements becomes effectively impossible because of a combinatorial explosion. This problem of exponential growth can only be accommodated by severely limiting the number of information units that are processed simultaneously. This is what learning processes such as schema construction and schema automation do by organising information in long-term memory. Schemas can be constructed during the problem-solving process by bringing elements together (i.e. chunking), by incorporating new elements in schemas already available in longterm memory or, more commonly, by obtaining already schematised information from other people. Schemas can then be treated as a single element in working memory and thus heavily decrease cognitive load associated with the performance of later tasks.

Constructed schemas may become automated if they are repeatedly applied and yield desired results. As is the case for schema construction, automation can free working memory capacity for other activities because an automated schema, acting as a central executive, directly steers behaviour without needing to process it in working memory. Because automation requires a great deal of practice, automated schemas only develop for those aspects of performance that are consistent across task situations, such as routines for operating medical equipment and standard procedures for using software applications. Thus, from an instructional design perspective, well-designed instruction should not only encourage schema construction, but should also support schema automation for those aspects that are consistent across tasks. ${ }^{6,11}$

\section{Types of cognitive load}

Novel information must be processed in working memory in order to construct schemas in long-term memory. The ease with which information may be processed in working memory is a focus of CLT. Working memory load may be affected by the intrinsic nature of the learning tasks (intrinsic load), by the manner in which the tasks are presented (extraneous load), and by the learning that actually occurs (germane load) when dealing with intrinsic load.

Intrinsic load cannot be altered by instructional interventions without altering the task to be learned (e.g. simplification) or by the act of learning itself. It depends on the number of elements that must be processed simultaneously in working memory, a number which, in turn, depends on the extent of element interactivity of the materials or tasks that must be learned. Element interactivity is the degree to which the elements of something to be learned can, or cannot, be understood in isolation. For example, vocabulary is an example of low element-interactive material in the field of language learning. Although there are thousands of words to be learned, most people can quickly learn some simple words because words may be learned in isolation from all other words. Grammar, by contrast, is an example of high element-interactive material. Most people have difficulty in generating grammatically correct sentences, even when all the words to be used in the sentence are known. This is because many elements must be considered simultaneously; that is, to build sentences that are grammatically correct, one must attend to all the words within the sentence at once while also considering syntax, tense and verb endings.

Tasks with high element interactivity are difficult to understand and yield a high cognitive load because learners must deal with several elements simultaneously. The only way to foster understanding and to reduce intrinsic cognitive load is to develop schemas that incorporate the interacting elements. It follows that a large number of interacting elements for one person might be included within a single element for another more experienced person who already has a schema that incorporates the elements. Thus, element interactivity can be estimated only by counting the number of interacting elements dealt with by people at a particular level of expertise.

By contrast with intrinsic load, extraneous load is imposed by instructional procedures. Extraneous load may be imposed, for example, when learners must use trial and error or other weak problemsolving methods that require them to arbitrarily try out things without being given proper guidance, ${ }^{12}$ when they must integrate information sources that are distributed in place or time, or when they must 
search for information that is needed to complete a learning task. Overloading one of the processors that constitute working memory may also increase it. Visual and auditory working memory are partially independent. If multiple sources of information that are required for understanding are all presented in visual form (e.g. a written text and a diagram), they are more likely to overload the visual processor than if the written material is presented in spoken form, thus enabling some of the cognitive load to be shifted to the auditory processor. ${ }^{13}$

Germane load, finally, refers to the working memory resources used to deal with intrinsic cognitive load, which lead to learning. For instance, learners construct schemas that deal with the interacting elements associated with intrinsic cognitive load by working on a series of tasks and abstracting away from them by identifying structural features and surface features in a process of induction or 'mindful abstraction'. They also construct schemas when they connect new information elements to the things they already know, that is, to existing schemas in long-term memory, in a process of elaboration. These processes of dealing with intrinsic cognitive load include elements related to previous tasks or to knowledge already available in long-term memory and thus require working memory resources that correspond to a germane cognitive load that is directly relevant for learning.

Cognitive load theory assumes that intrinsic and extraneous cognitive loads are additive. Whether extraneous load presents students with a problem depends, in part, on the intrinsic load. If intrinsic load is low, a high extraneous load resulting from an inadequate instructional design may not be harmful because the total cognitive load is within working memory limits. Indeed, research has shown that instruction designed to decrease extraneous load has negligible effects on learning simple tasks (i.e. involving low element-interactive materials $\left.{ }^{14}\right)$. There simply is no need to decrease extraneous load because there are sufficient cognitive resources available to deal with the low intrinsic cognitive load. However, for teaching complex tasks (i.e. involving high element-interactive materials), the sum of the intrinsic and extraneous loads may easily surpass working memory capacity and yield overload (Fig. 1a). Then, extraneous load and, if the reduction of extraneous load is still insufficient, intrinsic load must be lowered to free up processing resources necessary for learning (Fig. 1b). The more extraneous cognitive load is reduced, the more working memory resources can be devoted to intrinsic cogni-

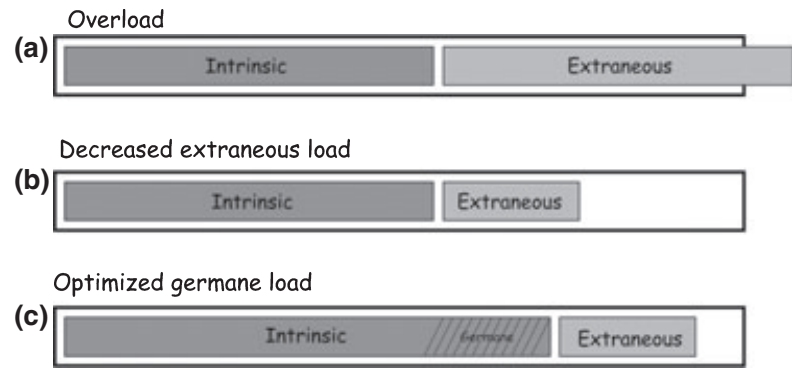

Figure 1 The additive nature of intrinsic and extraneous load: (a) overload; (b) preventing overload by decreasing extraneous load, and (c) optimising germane load by increasing intrinsic load

tive load and so the easier it becomes to induce a germane cognitive load for learning (Fig. 1c). The next section discusses design guidelines for managing cognitive load.

\section{DESIGN GUIDELINES}

Design principles and strategies based on CLT aim to prevent overload and optimise germane load in order to improve learning. The main principles and strategies for decreasing extraneous load, managing intrinsic load and optimising germane load are summarised and illustrated in Table 1. Moreover, they are related to the expertise reversal effect, which indicates that principles that work well for novice learners may not work well or may even have negative effects for more experienced learners.

\section{Decreasing extraneous load}

Sweller $e t a{ }^{2}{ }^{2}$ reviewed six research-based principles to decrease extraneous load for novice learners. Firstly, the goal-free principle suggests replacing conventional tasks with goal-free tasks, which provide learners with a non-specific goal (e.g. replace "find the most probable aetiological explanation for these symptoms' with 'find as many aetiological explanations for these symptoms as you can'). If learners are given a specific goal, they start reasoning from this goal and try to find operators that reduce the difference between the goal state and the given state. This backward search process yields a high extraneous load that can be avoided by eliminating a specific goal that renders working backward from the goal impossible.

Secondly, the worked example principle suggests replacing conventional tasks with worked examples that must be carefully studied by the learners. Because 
Table 1 Design principles and strategies recommended by cognitive load theory

\section{Design guideline Description}

Decreasing extraneous load

$\begin{array}{cc}\text { Goal-free } & \text { Replace conventional tasks with goal-free tasks } \\ \text { principle } & \text { that provide learners with a non-specific goal }\end{array}$

Worked example Replace conventional tasks with worked examples that principle

Completion principle provide a full solution learners must carefully study Replace conventional tasks with completion tasks that provide a partial solution learners must finish

Split attention principle

Modality

principle

dundancy principle

Replace multiple sources of information, distributed either in space (spatial split attention) or time (temporal split attention), with one integrated source of information

Replace a written explanatory text and another source of visual information (unimodal) with a spoken explanatory text and the visual source of information (multimodal)

Replace multiple sources of information that are self-contained (i.e. they can be understood on their own) with one source of information

Managing intrinsic load

Simple-to-complex Replace a series of conventional tasks with tasks that strategy

first present only isolated elements (low element interactivity) and gradually work up to the tasks in their full complexity

Low- to high-fidelity Replace a series of conventional tasks with tasks that strategy are first performed in a low-fidelity environment (decreased element interactivity), and then in increasingly higher-fidelity environments

\section{Optimising Germane load}

Variability

Replace a series of tasks with similar surface features principle with a series of tasks that differ from one another on

Contextual interference principle

Self-explanation principle all dimensions on which tasks differ in the real world Replace a series of task variants with low contextual interference with a series with high contextual interference

Replace separate worked examples or completion tasks with enriched ones containing prompts, asking learners to self-explain the given information

\section{Illustration}

Ask students to 'Please come up with as many illnesses as possible that could be related to the observed symptoms', rather than asking 'Which illness is indicated by the symptoms of this patient?'

Let students criticise a ready-made treatment plan, rather than having them independently generate such a plan

Let medical interns closely observe a surgical operation and only perform part of it, rather than having them perform the whole operation independently

Provide students with instructions for operating a piece of medical equipment just in time, precisely when they need it, rather than providing them with the information beforehand

Give students spoken explanations when they study a computer animation of the working of the digestive tract, rather than giving them written explanations on screen

When providing learners with a diagram of the flow of blood in the heart, lungs and body, do not include a verbal description of the flow

Give students tasks that require them to apply the basic physical principles of hydrodynamics, such as pressure-volume and pressure-flow relationships, before giving them tasks that require them to apply a full model of how the blood flows through the circulatory system When teaching students medical diagnosis, start with textual case descriptions, continue with computer-simu lated patients or patients played by peers, go on to simulated patients played by actors, and end with real patients in an internship in hospital

When describing a particular clinical symptom, illustrate it using patients of different sex, age, physique, medical history etc.

If students practise different variants of a particular surgical task, order these variants in a random rather than a blocked order

For students learning to diagnose malfunctions in the human cardiovascular system, present an animation of how the heart works and provide prompts that ask them to self-explain the underlying mechanisms 
Table 1 (Continued)

\section{Design \\ guideline \\ Description \\ Illustration}

Dealing with the expertise reversal effect

Completion Replace a uniform series of conventional tasks with a

strategy series of, in order, worked examples, completion tasks

for which learners must finish increasingly

larger parts of the solution, and conventional tasks

Fading

Replace a uniform series of conventional tasks with a

guidance

varying series of tasks that provide sizable learner

strategy

guidance in the beginning but gradually decrease

guidance until no guidance is given

(i.e. 'scaffolding')

Integrated to

Replace a uniform series of integrated examples with

non-integrated a two-stage series of integrated examples followed

strategy

by non-integrated examples, which present only the

picture or only the text

Dual- to

single-mode

Replace a uniform series of dual-mode presentations

strategy
For students learning to set up treatment plans, first let them study and criticise ready-made treatment plans, then let them finish partially completed treatment plans, and finally let them independently develop whole treatment plans

When students are learning to catheterise, first provide them with step-by-step instructions and feedback, then only provide them with feedback, and finally provide no guidance at all

Illustrate a new surgical procedure to medical students by presenting integrated pictures and text, but only use the pictures for experienced surgeons

Give students spoken explanations when they study a computer animation of the working of the digestive tract, but switch off the sound for more experienced students learners study rather than generate solutions, this reduces the high extraneous load caused by weak problem-solving methods. Thirdly, the completion principle suggests replacing conventional tasks with completion tasks, which provide a partial solution that must be completed by the learners. Like worked examples, completion tasks reduce extraneous load caused by weak problem-solving methods because giving part of the solution reduces the size of the problem space.

Fourthly, the split attention principle recommends replacing multiple sources of information that are distributed in space or time, such as pictures and accompanying text or a piece of equipment and instructions for operating it, with a single, integrated source of information (Fig. 2 ). The integrated information source reduces extraneous load because there is no need for the learners to mentally integrate the information from different sources.

Fifthly, the modality principle recommends replacing a written explanatory text and another source of visual information such as a diagram (unimodal) with a spoken explanatory text and the visual source of information (multimodal). Multimodal information presentation reduces extraneous load because it uses both the visual and the auditory processor of working memory and thus, in effect, increases available working memory capacity.

Sixthly, the redundancy principle suggests replacing multiple sources of information that are self-contained (i.e. they can be understood on their own) with only one source of information. The single source reduces extraneous load that is caused by unnecessarily processing redundant information.

\section{Managing intrinsic load}

For highly complex or ill-defined tasks, even after the removal of all sources of extraneous load, the element interactivity of the materials may still be too high to allow for learning. In such cases it becomes necessary to decrease intrinsic load. However, because intrinsic load is determined by the interaction between the nature of the materials being learned and the expertise of the learner, it cannot be altered without reducing learners' level of understanding. For learners to fully understand the 
(a)

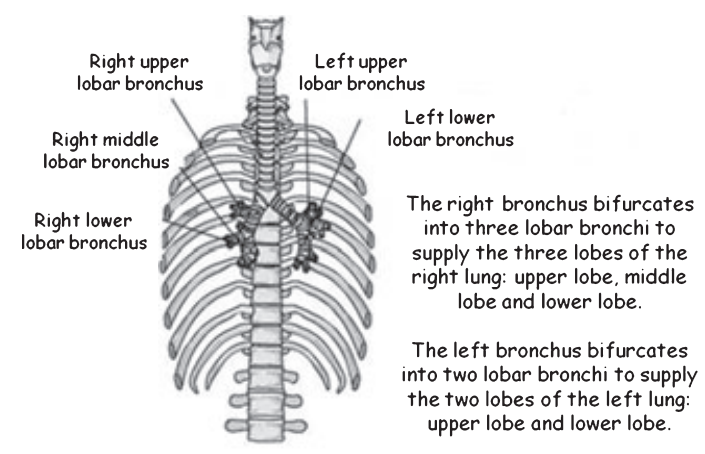

(b) Integrated format

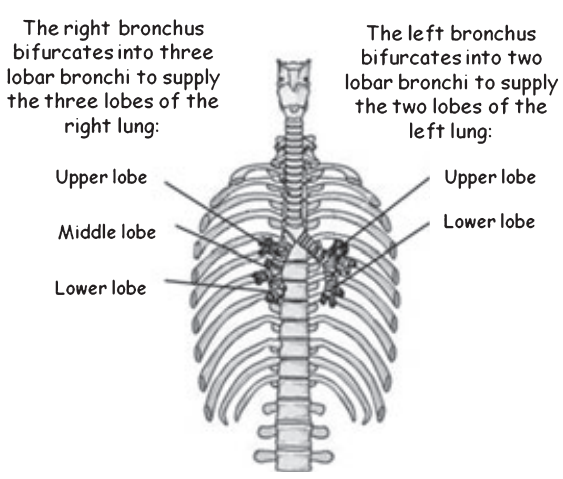

Figure 2 Text and picture in (a) a non-integrated format and (b) an integrated format

material, it must ultimately be presented in its full complexity. Multiple-step strategies rather than single-step principles are needed for sequencing materials from low to high element interactivity, so that tasks are presented in their full complexity only in a later learning phase.

Pollock et al. ${ }^{15}$ presented learners with a simple-tocomplex sequence of instruction. In the first part, intrinsic load was reduced by not presenting all the information at once. Instead, isolated elements that could be processed serially were presented. In the second part, however, all the information was presented at once, including the interactions among the elements. As hypothesised, although understanding was lower in the first phase of instruction when elements were presented in isolation, this deficiency was compensated in the second phase when the full set of interacting elements was presented. Thus, presenting the full set of interacting elements in both phases resulted in less understanding than presenting isolated elements in the first phase followed by the full set in the second phase.

Manipulating the fidelity of the learning environment is another way to gradually increase the number of interacting elements because high-fidelity environments will typically contain more interacting elements than low-fidelity environments. ${ }^{6}$ Although students of medicine, for example, may learn to diagnose ill-defined problems such as chronic low back pain immediately, they should not begin with real patients. It may be better to start with low-fidelity simulations (e.g. textual problems or case descriptions of prospective patients), then continue with medium-fidelity simulations (e.g. computer-simulated patients or simulated patients [SPs] played by peer students), continue with high-fidelity simulations (e.g. SPs played by actors), and end with the real environment (e.g. real patients during an internship in hospital; see example ${ }^{16}$ ).

\section{Optimising germane load by increasing intrinsic load}

The reduction of cognitive load prevents overload and frees up processing resources that can be devoted to genuine learning. It is sometimes desirable to increase intrinsic load in order to increase the associated germane load. Paas and van Merriënboer $^{17}$ were the first to relate variability of learning tasks to germane load. Variability of task situations encourages learners to construct cognitive schemas because it increases the probability that similar features can be identified and that relevant features can be distinguished from irrelevant ones. For example, when students learn about acid-base balance, different learning tasks should relate this concept to hyper- and hypoventilation, aspirin overdosing, loss of fixed acid through excessive vomiting, and so forth. Such exposure to a highly varied sequence of tasks or solutions helps learners determine the range of applicability of constructed schemas. Increasing variability increases intrinsic cognitive load. Students must learn which problems belong to the same or different categories, an important aspect of the task which is ignored under conditions of low variability.

Contextual interference is related to variability. It is low if adjacent tasks in a practice schedule rely on exactly the same skills and it is high if adjacent tasks rely on different skills. Van Merriënboer et al. ${ }^{18}$ compared a random practice schedule (e.g. BCBAABCCA) with high contextual interference with a blocked practice schedule (e.g. AAABBBCCC) with low contextual interference. As expected, high contextual interference increased intrinsic load by increasing germane load (i.e. including elements 
related to adjacent tasks) during practice and improved learning outcomes.

A third design principle is related to the so-called self-explanation effect. The intrinsic load and hence the germane load associated with explaining a concept to oneself are far higher than when simply processing the information because the number of interacting elements considered is deliberately increased by including prior-knowledge elements from long-term memory. Stark et al. ${ }^{19}$ studied learning from worked examples. They found that effective example elaboration is indeed associated with a higher germane load and improves learning outcomes.

\section{Expertise reversal effect}

An interaction effect that is particularly relevant when CLT is applied to the design of programmes with longer duration is the expertise reversal effect (see overview $^{20}$ ). This effect is an interaction between several basic cognitive load effects and level of expertise. The effect is demonstrated when instructional methods that work well for novice learners have no effects or even adverse effects when learners acquire more knowledge. Thus, multiple-step strategies are needed to sequence instructional methods in such a way that they take the growing level of expertise of learners into account.

Kalyuga et al..$^{21}$ demonstrated that, with increasing expertise, the worked example effect first disappears and then reverses. Worked examples become redundant for more knowledgeable learners and so impose an extraneous load. These findings suggest that a training programme should ideally start with worked examples and smoothly work up to conventional tasks. Van Merriënboer ${ }^{22}$ introduced the completion strategy to reach this goal: the training starts with full worked examples that include all of the solution steps, proceeds with a series of completion tasks that provide fewer and fewer of the required steps, and ends with conventional tasks that require learners to generate all required steps. Recent research indicates that the first phase can be further divided into one sub-phase in which students work on process-oriented worked examples and a second sub-phase in which they work on product-oriented examples. ${ }^{23}$

Renkl and Atkinson ${ }^{24}$ and Atkinson et al. ${ }^{25}$ reviewed studies that applied the completion strategy or alternative fading guidance procedures, where learner support and guidance decreased as learners acquired more expertise. They concluded that both the completion strategy and other fading guidance strategies are effective to manage cognitive load throughout the various phases of skill acquisition and yield higher learning outcomes than the traditional use of example-problem pairs.

The expertise reversal effect has also been found for the split attention principle and the modality principle. Kalyuga et al. ${ }^{26}$ demonstrated the usual split attention effect with novice learners. As levels of expertise increased, the advantage of the integrated conditions first disappeared and eventually reversed with the separate condition being superior to the integrated condition. The text had become redundant for experienced learners. Kalyuga $e t a l .{ }^{27}$ found that among novices, dual mode, auditory and visual presentations were superior to visual-only presentations, demonstrating the modality effect. With more experience, the auditory component became redundant and was best eliminated.

\section{CONCLUSIONS}

This article presents the cognitive architecture assumed by CLT and the main design guidelines that have been developed over the last 20 years. Because there is a close association between guidelines and cognitive architecture, we believe that applications of CLT yield effective instruction that is compatible with human cognitive processing. A rigorous testing programme, based on replicated and controlled experimental studies, has been characteristic of CLT. Current research in CLT concerns various questions related, for example, to the measurement of cognitive load, ${ }^{28,29}$ adaptive instruction ${ }^{30}$ and self-directed learning. ${ }^{31}$ Each of these research directions, along with many others, has the potential to change and improve CLT.

Contributors: both authors made substantial contributions to the study conception and design, and to the drafting and critical revision of the paper. Both authors approved the final manuscript for publication.

Acknowledgements: none.

Funding: none.

Conflicts of interest: none.

Ethical approval: not applicable.

\section{REFERENCES}

1 Sweller J. Cognitive load during problem solving: effects on learning. Cogn Sci 1988;12:257-85. 
2 Sweller J, van Merriënboer JJG, Paas F. Cognitive architecture and instructional design. Educ Psychol Rev 1998;10:251-96.

3 van Merriënboer JJG, Sweller J. Cognitive load theory and complex learning: recent developments and future directions. Educ Psychol Rev 2005;17:147-77.

4 Khalil MK, Paas F, Johnson TE, Payer AF. Interactive and dynamic visualisations in teaching and learning of anatomy: a cognitive load perspective. Anat Rec B New Anat 2005;286B:8-14.

5 Khalil MK, Paas F, Johnson TE, Su YK, Payer AF. Effects of instructional strategies using cross-sections on the recognition of anatomical structures in correlated CT and MR images. Anat Sci Educ 2008;1:7583.

6 van Merriënboer JJG, Kirschner PA. Ten Steps to Complex Learning: a Systematic Approach to Four-Component Instructional Design. Mahwah, NJ: Lawrence Erlbaum Associates 2007.

7 Kirschner PA, Sweller J, Clark RE. Why minimal guidance during instruction does not work: an analysis of the failure of constructivist, discovery, problem-based, experiential, and inquiry-based teaching. Educ Psychol 2006;41:75-86.

8 van Merriënboer JJG, Kester L, Paas F. Teaching complex rather than simple tasks: balancing intrinsic and germane load to enhance transfer of learning. Appl Cogn Psychol 2006;20:343-52.

9 Sweller J. Instructional implications of David C Geary's evolutionary educational psychology. Educ Psychol 2008;43:214-6.

10 Miller GA. The magical number seven, plus or minus two: Some limits on our capacity for processing information. Psychol Rev 1956;63:81-97.

11 van Merriënboer JJG, Kirschner PA, Kester L. Taking the load off a learner's mind: instructional design for complex learning. Educ Psychol 2003;38:5-13.

12 Anderson JR. Skill acquisition: the compilation of weakmethod problem solutions. Psychol Rev 1987;94:192210.

13 Mousavi S, Low R, Sweller J. Reducing cognitive load by mixing auditory and visual presentation modes. J Educ Psychol 1995;87:319-34.

14 Carlson R, Chandler P, Sweller J. Learning and understanding science instructional material. J Educ Psychol 2003;95:629-40.

15 Pollock E, Chandler P, Sweller J. Assimilating complex information. Learn Instruct 2002;12:61-86.

16 Maran NJ, Glavin RJ. Low- to high-fidelity simulation: a continuum of medical education? Med Educ 2003;37:22-8.

17 Paas F, van Merriënboer JJG. Variability of worked examples and transfer of geometrical problem-solving skills: a cognitive load approach. J Educ Psychol 1994;86:122-33.

18 van Merriënboer JJG, Schuurman JG, de Croock MBM, Paas F. Redirecting learners' attention during training: effects on cognitive load, transfer test performance, and training efficiency. Learn Instruct 2002;12:11-37.

19 Stark R, Mandl H, Gruber H, Renkl A. Conditions and effects of example elaboration. Learn Instruct 2002;12:39-60.

20 Kalyuga S, Ayres P, Chandler P, Sweller J. The expertise reversal effect. Educ Psychol 2003;38:23-31.

21 Kalyuga S, Chandler P, Sweller J, Tuovinen J. When problem solving is superior to studying worked examples. J Educ Psychol 2001;93:579-83.

22 van Merriënboer JJG. Strategies for programming instruction in high school: programme completion vs. programme generation. J Educ Comput Res 1990;6:26587.

23 van Gog T, Paas F, van Merriënboer JJG. Effects of studying sequences of process-oriented and productoriented worked examples on troubleshooting transfer efficiency. Learn Instruct 2008;18:211-22.

24 Renkl A, Atkinson RK. Structuring the transition from example study to problem solving in cognitive skill acquisition: a cognitive load perspective. Educ Psychol 2003;38:15-22.

25 Atkinson RK, Renkl A, Merrill MM. Transitioning from studying examples to solving problems: effects of selfexplanation prompts and fading worked-out steps. J Educ Psychol 2003;95:774-83.

26 Kalyuga S, Chandler P, Sweller J. Levels of expertise and instructional design. Hum Factors 1998;40:1-17.

27 Kalyuga S, Chandler P, Sweller J. Incorporating learner experience into the design of multimedia instruction. J Educ Psychol 2000;92:126-36.

28 DeLeeuw K, Mayer RE. Comparison of three measures of cognitive load: evidence for separable measures of intrinsic, extraneous, and germane load. J Educ Psychol 2008;100:223-34.

29 Paas F, Tuovinen JE, Tabbers H, van Gerven P. Cognitive load measurement as a means to advance cognitive load theory. Educ Psychol 2003;38:63-71.

30 Kalyuga S. Expertise reversal effect and its implications for learner-tailored instruction. Educ Psychol Rev 2007; 19:509-39.

31 van Merriënboer JJG, Sluijsmans DA. Towards a synthesis of cognitive load theory, four-component instructional design, and self-directed learning. Educ Psychol Rev 2008;21:55-66.

Received 4 February 2009; editorial comments to authors 4 March 2009; accepted for publication 8 July 2009 